\title{
Development and Application of Efficient Oil Displacement System for Middle-Low Permeability and High Pour-Point Heavy Oil Reservoirs
}

\author{
Zhenfu Ma, ${ }^{1,2}$ Kai Zhang ${ }^{\mathbb{D}},{ }^{1,3}$ Mengjie Zhao, ${ }^{1}$ Lu Liu, ${ }^{4}$ Chao Zhong, ${ }^{1}$ and Jian Wang ${ }^{5}$ \\ ${ }^{1}$ China University of Petroleum (East China), Qingdao, Shandong 266580, China \\ ${ }^{2}$ Hekou Oil Production Plant of SINOPEC Shengli Oilfield Company, Dongying, Shandong 257000, China \\ ${ }^{3}$ Qingdao University of Technology, Qingdao, Shandong 266580, China \\ ${ }^{4}$ Exploration and Development Research Institute of Dagang Oilfield Company, Tianjin 300280, China \\ ${ }^{5}$ Shengli Oil Field Exploration and Development Research Institute, Dongying, Shandong, 266071, China \\ Correspondence should be addressed to Kai Zhang; zhangkai@upc.edu.cn
}

Received 15 July 2021; Accepted 28 September 2021; Published 31 October 2021

Academic Editor: Wei Zhang

Copyright (C) 2021 Zhenfu Ma et al. This is an open access article distributed under the Creative Commons Attribution License, which permits unrestricted use, distribution, and reproduction in any medium, provided the original work is properly cited.

\begin{abstract}
In view of the problems of low permeability, high oil viscosity and freezing point, and low productivity of single well in Luo 321 and Luo 36 blocks of Luojia Oilfield, the chemical viscosity-reducing cold production technology was studied. By analyzing the properties of crude oil, it is concluded that the reason for high viscosity and high freezing point is the high content of asphaltene, pectin, and wax. The viscosity is mainly affected by asphaltene; the wax precipitation point and pour point are mainly affected by the wax; and the solidification point is affected by the wax and asphaltene. The treatment idea of reducing viscosity and inhibiting wax is determined. By compounding the synthetic pour point depressant POA-VA and the viscosity reducer DBD-DOPAMA, the effect of reducing the viscosity and freezing point of crude oil was evaluated. PD-7 (POA-VA 40\%, DBD-DOPAMA 50\%, and $\mathrm{P}-10 \mathrm{C} 10 \%$ ) system was selected as the optimal formula. When the concentration of the system is $10 \%$, the viscosity reduction rate reaches $95.2 \%$; the freezing point can reduce by $10.2^{\circ} \mathrm{C}$; it has good oil sample adaptability, salinity resistance, and temperature resistance; and the oil washing rate can reach more than $60 \%$. The oil displacement system was injected into the formation by means of multiconcentration and multislug and was applied in the field of Luo 321-2 Well. A total of 500 t of the oil displacement system was injected, and the effect of measures lasted for 400 days, with a cumulative oil increase of $883 \mathrm{t}$. It has been applied in different blocks 30 times and achieved a good field application effect.
\end{abstract}

\section{Introduction}

The middle-low permeability and high pour-point heavy oil reservoirs in Shengli Oilfield are widely distributed, with reserves of about 25 million tons, mainly distributed in Chengnan, Luojia-Kenxi, Wangiagang, Big D35-X20, Xin68, and other blocks. Taking Luo 321 and Luo 36 blocks of Luojia Oilfield in Hekou Oil Production Company as examples, the reservoir burial depth is more than $1600 \mathrm{~m}$, and the reservoir permeability is less than $300 \mathrm{mD}$. The viscosity of crude oil is greater than $50 \mathrm{mPa} \cdot \mathrm{s}$ at formation temperature (around $50^{\circ} \mathrm{C}$ ) and more than $50,000 \mathrm{mPa} \cdot \mathrm{s}$ at $20^{\circ} \mathrm{C}$, and the freezing point of crude oil is above $30^{\circ} \mathrm{C}$. At present, there are mainly the following problems in middlelow permeability and high pour-point heavy oil reservoirs. First, due to the dual influence of low permeability and thickened oil, the limit drainage radius of underground crude oil is small, and the productivity of a single well is low. Second, the reservoir is deep-buried, low permeability, high steam injection pressure, low enthalpy, and small heating radius. Third, high wax content of crude oil, high freezing point, wellbore lifting, and crude oil gathering and transportation is difficult. Therefore, conventional development and steam stimulation cannot achieve ideal results; reserves production rate, oil recovery speed, and development benefits are in a relatively low state. 
At present, by injecting viscosity reducer into the bottom of the well to reduce the viscosity of heavy oil and improve the flow of crude oil, this chemical viscosity reduction and cold production injection craft is gradually becoming an effective supplement to heavy oil steam injection production technology and has achieved certain application effect in the field $[1,2]$. However, this technology also has some limitations, the indoor viscosity reduction effect is significant, the field oil increase effect is very unstable, especially for hypercoagulability and high viscosity heavy oil; a single agent component is not targeted; and it is difficult to achieve the purpose of field oil increase [3-7]. This article selects Luo 321 and Luo 36 in Luojia oilfield as a test block. Based on the understanding of the causes of high pour point and high viscosity of crude oil, specific coagulants and thickeners have been developed according to the characteristics of crude oil.Through compatibility, a high-efficiency composite flooding system for low-permeability and high-pour-point heavy oil reservoirs is formed, and typical wells are selected for on-site application on the basis of indoor evaluation. It can solve the problems of reservoir flow, wellbore lifting, and ground oil collecting in an integrated way and provide strong technical support for the efficient development of similar reservoirs.

\section{Experimental Materials and Methods}

2.1. Materials and Instruments. Octadecyl acrylate (POA), vinyl acetate (VA), azo-iso-butyronitrile (AIBN), dopamine (DOPA), methacrylate chloride (MA), sodium borate, triethylamine are of analytical grade (all from Shanghai Aladdin Reagent Company); hydrochloric acid, sodium borate, methanol, $\mathrm{NaCl}, \mathrm{KCl}, \mathrm{Na} 2 \mathrm{SO} 4$ was from Sinopharm Chemical Reagent Co., Ltd; degassed heavy oil was from Luo 321 and Luo 36 blocks.

LP1002 Electronic Balance was from Changshu Weighing Device Factory; CH702 constant temperature stirred water bath was from Gongyi Yuhua Instrument Co., Ltd.; Agilent 6890 GC gas chromatograph was from Germany IKA ; Quartz crystal microbalance was from Biolin Sweden Company; DHG-9070A electric heating blast drying oven was from Shanghai Yiheng Scientific Instrument Co.,
Ltd. DV-II + PRO viscometer was from USA Brookfield Company; the atomic force microscope (AFM) was used.

2.2. Determination of Crude Oil Properties. The standard SH/ T 0509-2010 "determination of four components of petroleum asphalt," SY/T 0521-2008 “crude oil wax point determination of microscopic observation method," SY/T 7516-2010 "modified crude oil pour point determination of melting method," and SY/T 0541-2009 "crude oil condensation point determination method" were used to determine the four components, wax extraction point, pour point, and freezing point of crude oil in this area, respectively.

2.3. Crude Oil Viscosity-Temperature Relationship Test. After preheating the sample to $60^{\circ} \mathrm{C}$, it was placed in the rotary viscometer measurement system. After the temperature stabilized, the sample was cooled at a rate of $0.2-0.9^{\circ} \mathrm{C} /$ $\min$. The flat plate test system was used to record the changes of shear stress and viscosity with the change of temperature at the shear rate of $5 \mathrm{~S}^{-1}$, and the curve was drawn.

2.4. Full Component Analysis of Carbon Group of Crude Oil. Agilent 6890 GC gas chromatogram was used to test the total carbon components of crude oil and determine the peak value of carbon components. PONA quartz capillary column was used for the chromatographic column with a length of $50 \mathrm{~mm}$ and an inner diameter of $0.20 \mathrm{~mm}$. The detector used hydrogen flame ionization detector, $220^{\circ} \mathrm{C}$; the temperature of the vaporization chamber is $200^{\circ} \mathrm{C}$; the temperature of the column is $80^{\circ} \mathrm{C}-320^{\circ} \mathrm{C}$; and the temperature change rate is $6^{\circ} \mathrm{C} / \mathrm{min}$. The flow rate of hydrogen is $40 \mathrm{ml} / \mathrm{min}$, and that of air is $400 \mathrm{ml} / \mathrm{min}$.

2.5. Synthesis of POA-VA Pour Point Depressant. Totally, $32.4 \mathrm{~g}$ octadecyl acrylate (POA) and $8.6 \mathrm{~g}$ vinyl acetate (VA) were dissolved in $160 \mathrm{~g}$ mineral oil, and $0.3 \mathrm{~g}$ of initiator azoiso-butyronitrile (AIBN) was added under stirring condition; then $\mathrm{N}_{2}$ was passed through for $30 \mathrm{~min}$ for deoxygenation, and the reaction was heated at $85^{\circ} \mathrm{C}$ for 10 hours. The reaction equation is as follows:

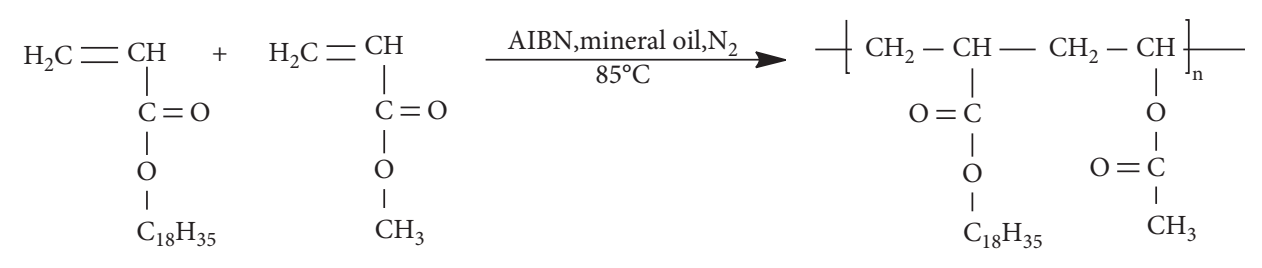

2.6. Synthesis of DBD-DOPAMA Viscosity Reducer. Dopama methacrylate (DOPAMA) was firstly synthesized by adding $\mathrm{DOPA} \bullet \mathrm{HCl}$ and methacrylate chloride (MA) into $10 \mathrm{~mL}$ methanol at a molar ratio of $1: 1$, adding sodium borate and triethylamine, and stirring at room temperature for 24 hours to get DOPAMA. The new viscosity reducer DBDDOPAMA can be obtained by polymerization of the synthesized DOPAMA with DBD. The reaction equation is as follows: 




2.7. Oil Washing Ability Test. Quartz crystal microbalance was used to determine the oil absorption capacity (QCM-D) of PD-7. The experimental procedures are as follows (Figure 1):

(i) The heavy oil of Luo321-2 was quantitatively adsorbed on the surface of the quartz test sheet, and the mass was weighed $m_{1}$.

(ii) The heavy oil has continued to be cleaned on the surface of the quartz slice with a $10 \%$ concentration PD-7 solution. After the detector signal is stable, the quartz slice was taken out, and the mass was weighed $m_{2}$, and the oil washing rate $D=100 \% \times\left(m_{1}-m_{2}\right) / m_{1}$.

(iii) The surface of the peeled quartz was then mapped using atomic force microscopy (AFM).

\section{Experimental Results and Discussion}

\subsection{Cause Analysis of High Freezing Point and High Viscosity of Heavy Oil}

3.1.1. Composition and Property Analysis of Crude Oil. Crude oils from six wells in Luojia Oilfield, including Luo 321-1, Luo 321-2, Luo 36-8, Luo 36-9, Luo 36-25, and Luo $32-1$, were measured for their composition, wax extraction points, and other properties. The experimental data are shown in Table 1, and the viscose-temperature relationship was tested. The experimental results are shown in Figure 2. The experimental results show that the viscosity, wax precipitation point, pour point, and freezing point are highly related to the content, composition, and ratio of heavy components. The higher the molecular weight of asphaltenes, gums, and waxes, the stronger the interaction between the heavy components with an increase of the viscosity, wax precipitation point, pour point, and freezing point, and vice versa. When the wax molecular weight is C25 and the wax content is more than $20 \%$, the wax crystal aggregates can be completely dispersed when the temperature is above $70^{\circ} \mathrm{C}$, and the wax precipitation point is mainly affected by the wax. When the molecular weight of asphaltene is C25 and the content is more than $20 \%$, the crude oil loses its fluidity when the temperature drops to $40^{\circ} \mathrm{C}$, and the viscosity, pour point, and freezing point are mainly affected by asphaltene. When the molecular weight of the heavy component is $\mathrm{C} 13$ and the colloid content is more than $20 \%$, the viscosity is mainly affected by the colloid. The freezing point is mainly affected by the wax. At the same time, the colloid can inhibit the aggregation of wax crystals and reduce the wax extraction point and pour point.

3.1.2. Mechanism of Action of High Condensation, High Viscosity, and Heavy Oil Components. Study shows that the high viscosity of heavy oil is due to its composition contains high content of colloid and asphaltene. When there is no wax, the asphaltenes mainly form a layered accumulation structure through $\pi-\pi$ bonds, and the colloids are adsorbed and wound around the asphaltene aggregates through van der Waals force and electrostatic action, isolating asphaltene aggregates of various sizes. When the temperature drops, the thermal motion of the molecules decreases. In such a situation, aggregate spacing shortens and interaction intensifies, leading to that the viscosity of crude oil increases greatly [8]. The asphaltene aggregates have little influence on the freezing point and pour point of crude oil due to the fact that the asphaltene aggregates can slide between layers, and there is a certain compaction space between layers [9].

But when the wax content in the crude oil is higher, the situation is different. GROMACS software is adopted to simulate the interaction between wax and asphaltene molecules, as shown in Figure 3.After the wax and asphaltene plastic wrap (Figures 3(b) and 3(c)), the high tension of the former long carbon chain caused the conjugate surface bent of the latter aromatic ring (Figure 3(e)) and weakened the $\pi-\pi$ connection. As a result, part of the asphaltene shifted from the plane-to-surface layered structure to the internal cluster structure of the asphaltene binding wax molecules (Figure 3(d)), and the volume of the dispersed asphaltene clusters increased significantly (Figure 3(f)), that is, waxcoated asphaltene clusters. The asphaltene is no longer stacked in layers, and the volume of the clusters formed increases greatly; the distance between asphaltenes decreases; and the movement space decreases, resulting in the increase of the freezing point and pour point of crude oil.

Through the above analysis, it is found that the viscosity of high condensation and high viscous oil is mainly affected by asphaltene, the wax extraction point and pour point are mainly affected by wax, and the freezing point is affected by both of them. When the viscosity is higher, it will show the characteristics of heavy oil, and when the viscosity is lower, it will show the characteristics of high pouching oil [10]. Therefore, a single functional agent cannot solve the problems of high pouching and high viscosity at the same time, and the wax suppression must be coordinated with the viscosity reduction. 


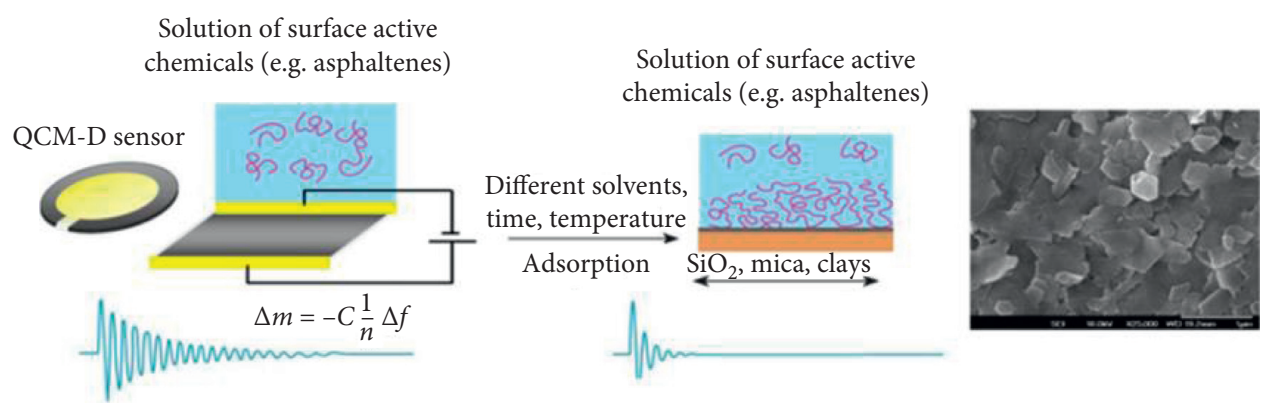

FIGURE 1: The working principle of quartz crystal microbalance.

TABLE 1: Four component analysis results of crude oil.

\begin{tabular}{|c|c|c|c|c|c|c|c|c|c|}
\hline \multirow[b]{2}{*}{ Sample ID } & \multicolumn{5}{|c|}{ Component analysis } & \multicolumn{4}{|c|}{ Characteristic analysis } \\
\hline & $\begin{array}{c}\text { Alkane } \\
(\%)\end{array}$ & $\begin{array}{l}\text { Arene } \\
(\%)\end{array}$ & $\begin{array}{l}\text { Pectin } \\
(\%)\end{array}$ & $\begin{array}{l}\text { Asphaltene } \\
(\%)\end{array}$ & $\begin{array}{c}\text { Waxiness } \\
(\%)\end{array}$ & $\begin{array}{c}\text { Wax precipitation } \\
\text { point }\left({ }^{\circ} \mathrm{C}\right)\end{array}$ & $\begin{array}{c}\text { Pour } \\
\text { point } \\
\left({ }^{\circ} \mathrm{C}\right)\end{array}$ & $\begin{array}{l}\text { Freezing } \\
\text { point }\left({ }^{\circ} \mathrm{C}\right)\end{array}$ & $\begin{array}{c}\text { Peak carbon } \\
\text { composition of } \\
\text { crude oil }\end{array}$ \\
\hline Luo 321-1 & 25.19 & 11.83 & 18.22 & 24.43 & 20.33 & 69.1 & 50.3 & 40.3 & $\mathrm{C} 25$ \\
\hline Luo $321-2$ & 16.78 & 10.40 & 17.52 & 25.82 & 29.48 & 72.5 & 52.1 & 41.8 & $\mathrm{C} 13$ \\
\hline $\begin{array}{l}\text { Luo } 36- \\
\text { oblique } 25\end{array}$ & 19.64 & 16.66 & 12.63 & 22.96 & 18.11 & 62.2 & 50.1 & 39.2 & $\mathrm{C} 25$ \\
\hline $\begin{array}{l}\text { Luo } 36- \\
\text { oblique } 8\end{array}$ & 15.08 & 18.36 & 25.49 & 16.86 & 14.21 & 50.3 & 38.1 & 27.3 & $\mathrm{C} 13$ \\
\hline $\begin{array}{l}\text { Luo } 36- \\
\text { oblique } 9\end{array}$ & 18.08 & 19.27 & 24.23 & 12.61 & 15.81 & 49.8 & 39.1 & 28.8 & $\mathrm{C} 25$ \\
\hline Luo $32-1$ & 14.80 & 23.08 & 21.84 & 19.18 & 11.10 & 51.2 & 41.1 & 29.3 & $\mathrm{C} 25$ \\
\hline
\end{tabular}

3.2. Formulation Optimization and Performance Evaluation of High-Efficiency Oil Displacement System with Pour Point and Viscosity Reduction

3.2.1. Compound of the Efficient Oil Displacement System. Through literature research, POA-VA and DBD-DOPAMA were synthesized in our laboratory. The two agents were mixed to screen the system of pour-point and viscosity reduction, and a total of 9 different mixing ratios were screened. At the same time, surfactant P-10C was added to improve the miscible effect. As shown in Figure 4, the higher the mass ratio of POA-VA, the more inclined the mixed solution was to water-in-oil emulsion; on the contrary, the higher the mass ratio of DBD-DOPAMA, the more inclined the mixed solution was to a water-in-oil emulsion, and the compatibility of the two were good are shown in Table 2.

3.2.2. Pour-Point Reduction and Viscosity Reduction Tests of Efficient Oil Displacement Composite System. (1) Viscosity reduction energy test of the compound system: According to the method specified in Q/SH1020 1519-2016 "General technical specification for heavy oil viscosity reducer," taking Luo 321-2 heavy oil with the highest viscosity as the benchmark, the viscosity reduction rate of pour point and viscosity reduction compound agent was tested, and the results are shown in Table 3.

As can be seen from Table 3, pure pour point depressor depressant POA-VA (system 1) basically has no viscosity reduction effect, and the reduction effect rate of the complex system 2-4 is not up to the standard because the content of the viscosity reduction agent DBD-DOPAMA is less than $40 \%$, resulting in insufficient viscosity reduction efficiency. The viscosity reduction rates of the complex systems 5-10 can all reach more than $90 \%$, and the viscosity reduction rates of systems 7, 8, and 9 are the highest. The reason is that the wax crystals released from the asphaltene after viscosity reduction are destroyed by the wax inhibitor, which reduces the interaction between asphaltene wax-gum and increases the viscosity reduction rate.

(2) Pour point reduction performance test of the compound system: Taking the freezing point and Luo 321-2 heavy oil with the highest wax content as the benchmark, system 5-11 with better viscosity reduction effect was selected to test the freezing point reduction effect. The experimental results are shown in Table 4.

As can be seen from Table 4, pure viscous-reducing agent DBD-DOPAMA (system 11) basically has no effect on reducing the freezing point. As the proportion of POA-VA in the recombination increases, the freezing point reduction range increases first and then decreases. The combination system 6-8 has the best effect of relatively reducing the freezing point because viscosity reduction and paraffin suppression make most of the asphaltenes arranged in layers instead of clusters, which improves the movement space and effectively reduces the freezing point. System 7 reduces the freezing point to $10.2^{\circ} \mathrm{C}$.

(3) PD-7 adaptability test: After comprehensive consideration of the viscosity reduction and pour-reducing effects of the complex system as well as the price factors, it is finally determined that complex system 7 is the optimal 




Figure 2: Viscosity-temperature curve of each oil sample.
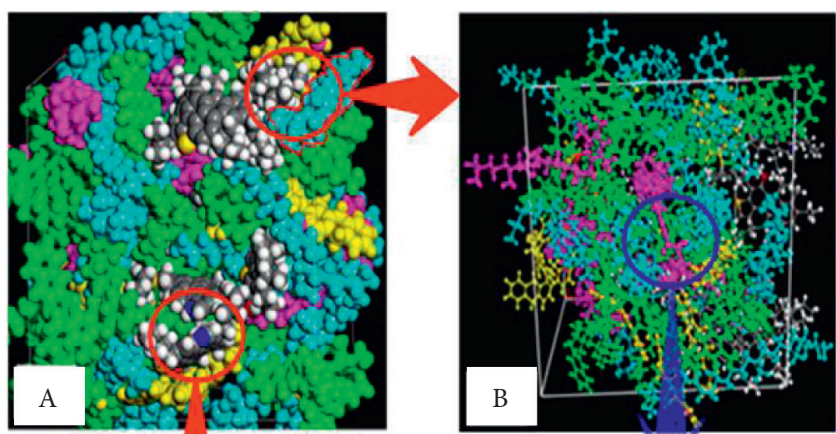

asphaltene 1

asphaltene 2

asphaltene 3

gelatine 1

gelatine 2

waxiness 1

waxiness 2

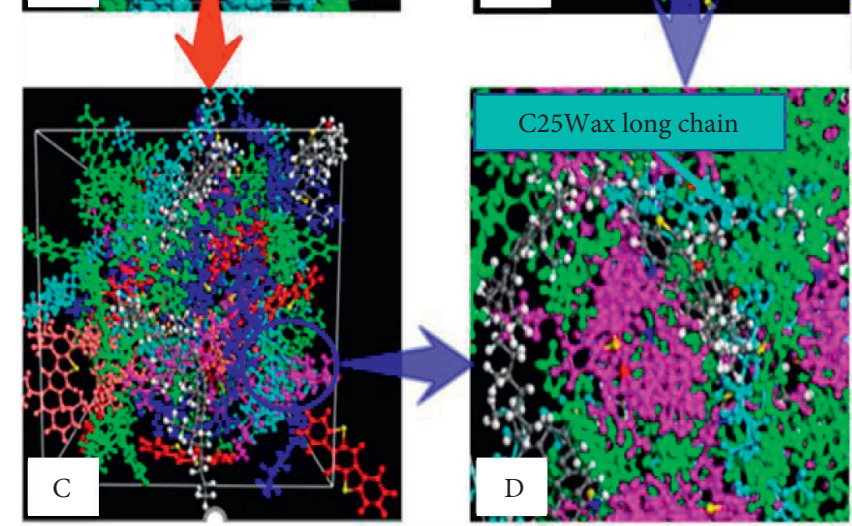

waxiness 3
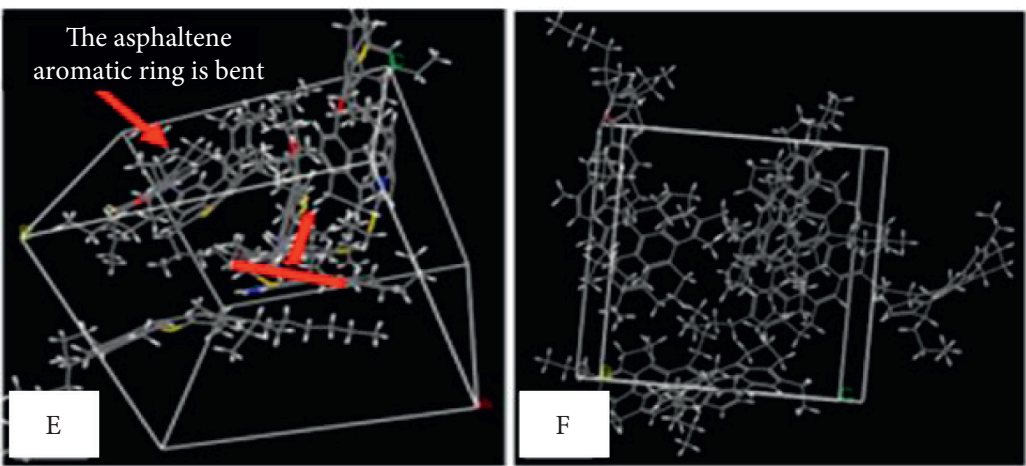

FIGURE 3: GROMACS software simulated the molecular interaction between wax and asphaltene. 




FIgURE 4: Complex viscosity reducing and wax inhibiting system.

TABLE 2: Compound viscosity reducing and wax suppression system.

\begin{tabular}{lccccccccccc}
\hline $\begin{array}{l}\text { Compound } \\
\text { components }\end{array}$ & System 1 & System 2 & System 3 & System 4 & System 5 & System 6 & System 7 & System 8 & System 9 & $\begin{array}{c}\text { System } \\
10\end{array}$ & $\begin{array}{c}\text { System } \\
11\end{array}$ \\
\hline POA-VA & 100 & 80 & 70 & 60 & 50 & 45 & 40 & 30 & 20 & 10 \\
DBD-DOPAMA & 0 & 10 & 20 & 30 & 40 & 45 & 50 & 60 & 70 & 80 & 100 \\
P-10C & 0 & 10 & 10 & 10 & 10 & 10 & 10 & 10 & 10 & 10 & 0 \\
\hline
\end{tabular}

TABLE 3: The effect of the compound of reducing viscosity and inhibiting wax on reducing viscosity.

\begin{tabular}{lccccccccccc}
\hline $\begin{array}{l}\text { Drug concentration, } \\
\text { (mOil:mPotion = 1:1) }\end{array}$ & System & System & System & System & System & System & System & System & System & System & System \\
& 1 & 2 & 3 & 4 & 5 & 6 & 7 & 8 & 9 & 10 & 11 \\
\hline 0 & 0 & 0 & 0 & 0 & 0 & 0 & 0 & 0 & 0 & 0 & 0 \\
2 & 2.1 & 42.4 & 48.4 & 65.1 & 80.3 & 82.2 & 88.1 & 89.8 & 88.3 & 86.4 & 82.4 \\
5 & 5.2 & 58.1 & 68.1 & 75.1 & 85.5 & 88.5 & 92.5 & 93.3 & 91.8 & 89.8 & 88.1 \\
10 & 8.1 & 61.3 & 72.1 & 80.3 & 90.2 & 92.2 & 95.2 & 96.7 & 94.3 & 92.5 & 91.3 \\
\hline
\end{tabular}

TABLE 4: Effect of viscosity reducing and wax inhibiting compound on freezing point.

\begin{tabular}{lccccccc}
\hline Drug concentration, \% (mOil:mPotion = 1:1) & System 5 & System 6 & System 7 & System 8 & System 9 & System 10 & System 11 \\
\hline 0 & 41.8 & 41.8 & 41.8 & 41.8 & 41.8 & 41.8 & 41.8 \\
2 & 40.1 & 39.3 & 38.3 & 38.7 & 39.5 & 40.3 & 41.3 \\
5 & 38.1 & 37.8 & 35.8 & 36.8 & 38.8 & 39.1 & 40.8 \\
10 & 34.7 & 32.8 & 31.6 & 33.1 & 36.6 & 38.4 & 40.1 \\
\hline
\end{tabular}

formula combination (PD-7). The concentration of $2-10 \%$ has the effect of viscosity reduction and pour-reducing, and the viscosity reduction rate reaches $95.2 \%$ at the concentration of $10 \%$. The measured wax extraction point, pour point, and freezing point can be reduced to $13.7^{\circ} \mathrm{C}, 17.4^{\circ} \mathrm{C}$, and $10.2^{\circ} \mathrm{C}$, respectively. As a slug reservoir agent, it can effectively reduce viscosity, improve oil flow capacity, avoid the risk of wax extraction and wax formation and enable oil to be pumped smoothly and in normal production. The adaptability test of this system in other oil samples was carried out, and the results are shown in Table 5 . The reason for choosing $50^{\circ} \mathrm{C}$ chosen to test of viscosity reduction rate is that $50^{\circ} \mathrm{C}$ is the most representative and closer to the actual formation temperature. The viscosity reduction rate of $\mathrm{PD}-7$ with $10 \%$ concentration on the oil samples was all over $95 \%$, and the wax extraction point, pour point, and freezing point were all reduced by more than $10^{\circ} \mathrm{C}$. That is to say, $\mathrm{PD}-7$ has a universal viscosity reduction and paraffin suppression effect on the low permeability and high condensing heavy oil in Luo321 and Luo36 blocks.

3.2.3. Performance Test of Efficient Oil Displacement System $P D-7$. (1) Resistance to salinity performance: $\mathrm{NaCl}, \mathrm{KCl}$, and $\mathrm{Na} 2 \mathrm{SO} 4$ were used to simulate formation water, and PD-7 solutions of $5 \%$ and $10 \%$ were prepared with water of different salinity degrees, respectively, to evaluate the influence of salinity on the viscosity reduction rate and freezing point of PD-7. The test temperature is set to $50^{\circ} \mathrm{C}$, and the experimental results are shown in Figure 5.

As can be seen from Figure 5, the viscosity reduction rate increased slightly with the increase of salinity, while the freezing point decreased slightly. When the salinity increased by $100,000 \mathrm{ppm}$, the viscosity reduction rate of Luo 321-2 heavy oil with $10 \%$ PD-7 concentration was $96.3 \%$, and the freezing point decreased by $10.6^{\circ} \mathrm{C}$. This is because 
TABLE 5: Adaptability test for viscosity reduction and wax suppression of the compound system 7 .

\begin{tabular}{|c|c|c|c|c|c|c|c|c|c|c|}
\hline \multirow{2}{*}{$\begin{array}{l}\text { Drug concentration, } 10 \% \text { (mOil: } \\
\text { mPotion }=1: 1 \text { ) }\end{array}$} & \multirow{2}{*}{$\begin{array}{l}\text { Viscosity ratio }(\%, \\
\left.50^{\circ} \mathrm{C}\right)\end{array}$} & \multicolumn{3}{|c|}{$\begin{array}{l}\text { Wax precipitation point } \\
\qquad\left({ }^{\circ} \mathrm{C}\right)\end{array}$} & \multicolumn{3}{|c|}{ Pour point $\left({ }^{\circ} \mathrm{C}\right)$} & \multicolumn{3}{|c|}{ Freezing point $\left({ }^{\circ} \mathrm{C}\right)$} \\
\hline & & Beginning & Dosage & $\Delta$ & Beginning & Dosage & $\Delta$ & Beginning & Dosage & $\Delta$ \\
\hline$\overline{\text { Luo } 3}$ & 96.8 & 69.1 & 55 & -14.1 & 50.3 & 33.7 & -16.6 & 40.3 & 29.2 & -11.1 \\
\hline Luo 3 & 9 & 50.3 & 39.1 & -11.2 & 38.1 & 22.8 & -15.3 & 27.3 & 17.1 & -10.2 \\
\hline Luo 36 - oblique 9 & 96.2 & 49.8 & 37 & -12.8 & 39.1 & 24.5 & -14.6 & 28.8 & 18.1 & -10.7 \\
\hline Luo 36 - oblique 25 & 98.1 & 62.2 & 48.4 & -13.8 & 48.7 & 30.6 & -18.1 & 39.2 & 26.9 & -12.3 \\
\hline Luo $32-1$ & 96.3 & 51.2 & 39.1 & -12.1 & 41.1 & 27 & -14.1 & 29.3 & 18.5 & -10.8 \\
\hline
\end{tabular}

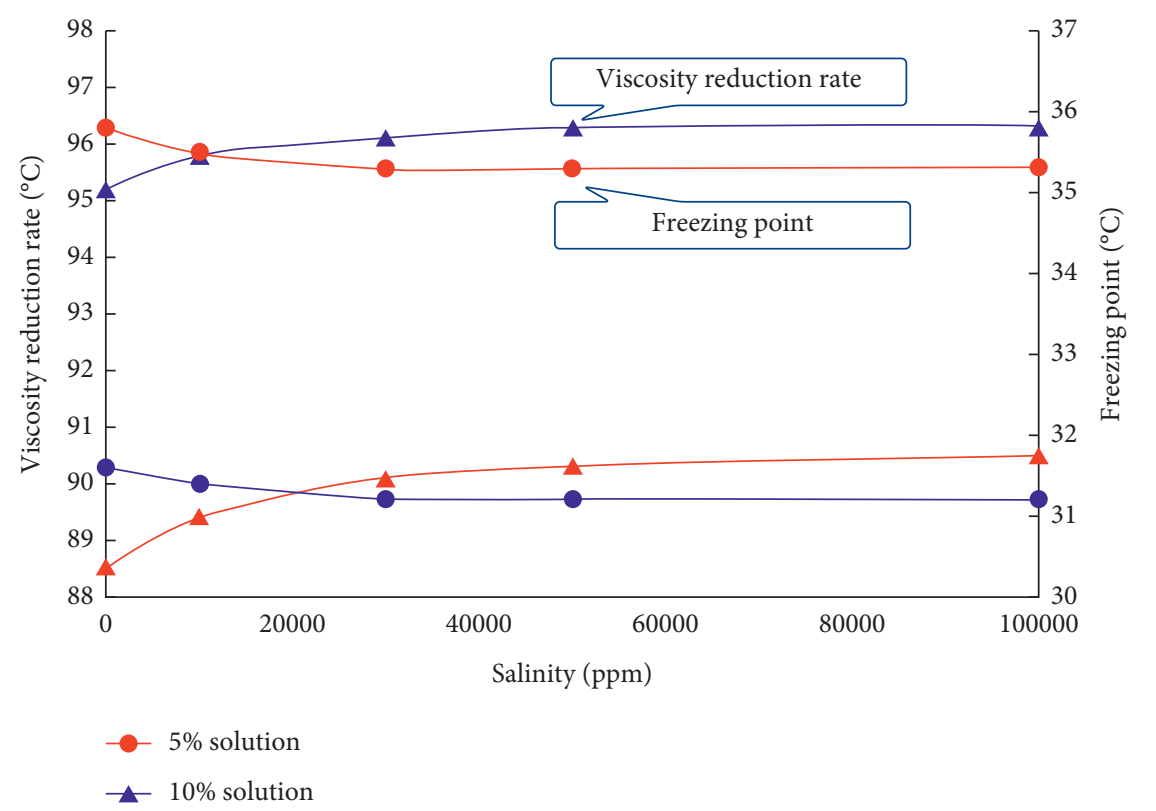

FIGURE 5: Influence of salinity on viscosity reduction rate and freezing point of PD-7.

the cation concentration increases at high salinity, and the ionized cation significantly increases the polarity of ortho dihydroxyl groups, further improves the polarity of catechol groups, increases the interfacial activity of DBD-DOPAMA, and enhances the emulsification effect, which is conducive to improving the viscosity reduction rate.

(2) Temperature resistance: The influence of $5 \%$ and $10 \%$ PD-7 solution on the viscosity reduction rate and freezing point of crude oil was measured at different temperatures, and the influence of temperature on the viscosity reduction rate and freezing point of PD-7 was evaluated. The experimental results are shown in Table 6.

It can be seen from Table 6 that when the temperature is higher than $100^{\circ} \mathrm{C}$, the viscosity of luo321-2 heavy oil is less than $200 \mathrm{MPa} \cdot \mathrm{s}$, the viscosity after viscosity reduction is less than $30 \mathrm{MPa} \cdot \mathrm{s}$, and the viscosity of light oil with API $>31.1$ is basically $10-35 \mathrm{MPa} \cdot \mathrm{s}$. That is to say, there is no problem in the fluidity after viscosity reduction above $100^{\circ} \mathrm{C}$. In fact, the effect at this time is to improve the fluidity rather than viscosity reduction, and the corresponding viscosity reduction rate is no longer of practical significance. Therefore, PD-7 can maintain its activity at $130^{\circ} \mathrm{Cwashing}$ oil ability.

The oil washing ability of $0.5 \%, 2 \%, 5 \%$, and $10 \%$ PD-7 solution was evaluated, and the experimental results were shown in Figure 6. The red line represents that the QCM-D detection signal is stable, that is, the oil washing is over, and the remaining crude oil adsorbed on the quartz surface cannot be further stripped.

According to the results of QCM-D, a curve was plotted (see Figure 7). As can be seen from the curve, with the increase of PD-7 concentration, the oil washing rate also increases. When the concentration reaches $10 \%$, the oil washing rate is $>60 \%$; at this point, the washing rate tends to be stable.

AFM is used to map the remaining oil distribution of quartz slices, and the experimental results are shown in Figure 8 . With the increase of PD-7 concentration, the area of a bright spot on quartz surface increases, and the stripping effect of crude oil increases. When $\mathrm{C}=0.5 \%, \mathrm{PD}-7$ still has a certain stripping ability to the crude oil on the surface of the sandstone. When $\mathrm{C}=10 \%$, the crude oil is stripped and cleaned in a large area, but compared with $5 \%$, the oil washing ability tends to be stable, and the stripping effect of the remaining oil decreases with the increase of concentration. So if the oil near the well is thick and causes a blockage, it can be cleaned quickly with a high concentration of agents. When the injection pressure of heavy oil is too high in water injection and displacement, the concentration 
TABLE 6: Influence of different temperatures on viscosity reduction rate and freezing point of PD-7.

\begin{tabular}{lcccc}
\hline \multirow{2}{*}{ Temperature $\left({ }^{\circ} \mathrm{C}\right)$} & \multicolumn{2}{c}{ Viscosity reduction rate $(\%)$} & \multicolumn{2}{c}{ Freezing point $\left({ }^{\circ} \mathrm{C}\right)$} \\
& $\mathrm{C}=5 \%$ & $\mathrm{C}=10 \%$ & $\mathrm{C}=5 \%$ & 35.1 \\
$\mathrm{C}=10 \%$ \\
\hline 80 & 94.1 & 98.1 & 34.5 & 31.6 \\
90 & 95.1 & 98.8 & 34.3 & 30.4 \\
100 & 86.5 & 89.1 & 34.3 & 29.2 \\
120 & 77.4 & 80.1 & 34.3 & 29.2 \\
130 & 50.1 & 60.5 & 29.2 \\
\hline
\end{tabular}
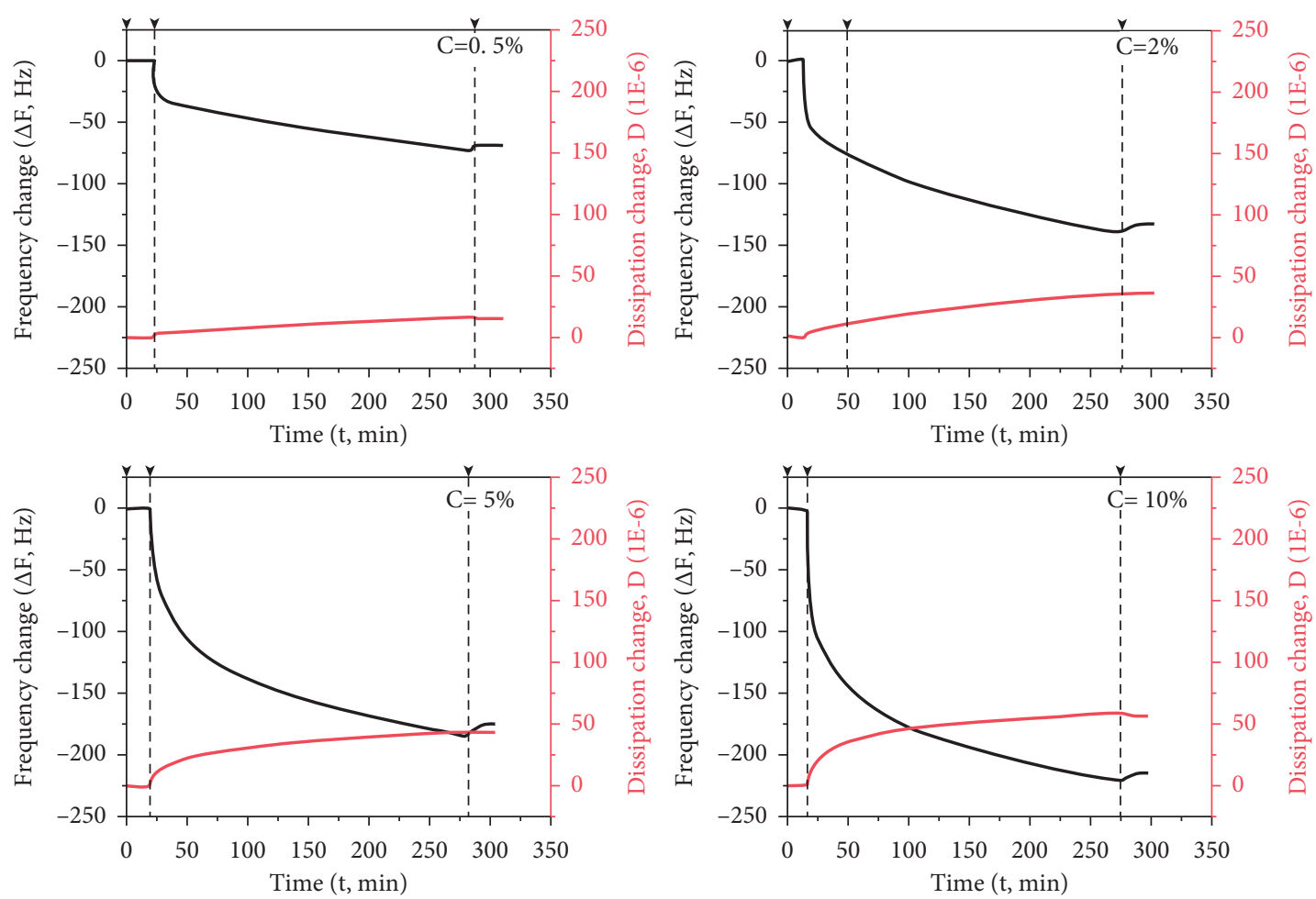

Figure 6: QCM-D results of different concentrations of PD-7.

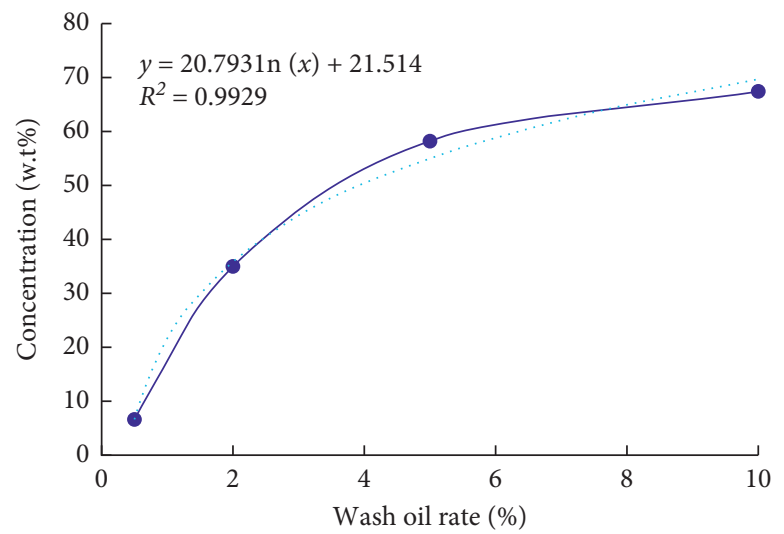

FIGURE 7: Relationship between different concentrations of PD-7 and oil washing rate. 



FIGURE 8: Stripping effect of heavy oil with different concentrations of PD-7.

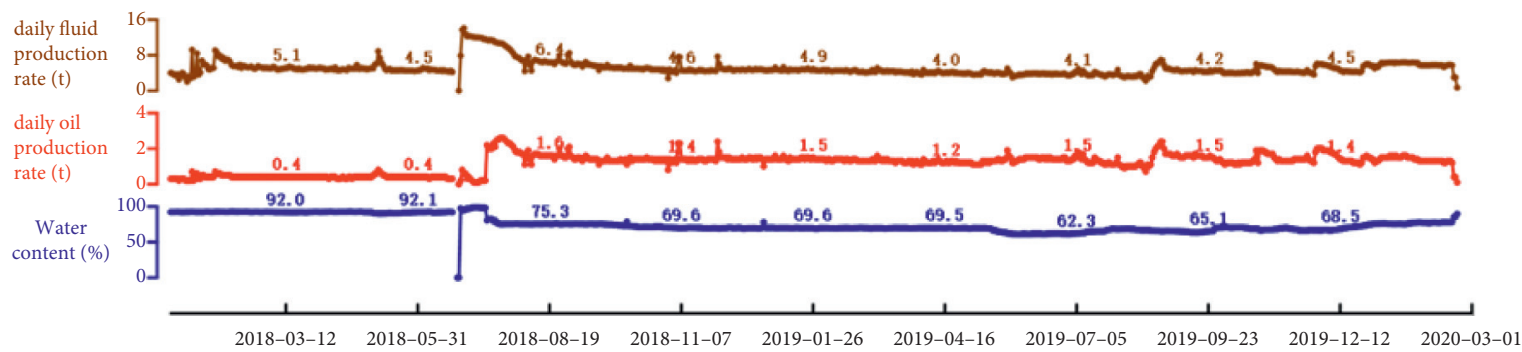

FIGURE 9: Oil production curve of well Luo 321-2.

can be increased in time to clean the oil wall area formed through the reservoir, so as to avoid the invalid injection caused by the change of injection direction.

\subsection{Reservoir Test Application}

3.3.1. Injection Process. Three slug concentration injection method was used: the first slug is injected with high displacement (20-40 t/h) and high concentration (8-10\%) oil displacement system to reduce viscosity and pour point; remove the wax crystal, gum, and asphaltene retained on the rock surface; solve the problem of organic matter blockage near the well zone; and avoid excessive dilution of subsequent injection agent to reduce the effect. In the second slug, high displacement $(20-40 \mathrm{t} / \mathrm{h})$ is injected into the medium concentration (5-8\%) displacement system to clean the reservoir, improve the oil production channel, and improve the reservoir permeability. In the third slug fluid, low displacement $(10-20 \mathrm{t} / \mathrm{h})$ is injected into low concentration (2-5\%) displacement system to improve the sweep range of injection agent, enhance the mobility of heavy oil in the early high-speed injection space, and improve the liquidity of crude oil and the connectivity of reservoir energy.

3.3.2. Field Application. Luo 321-2 well is preferred for field implementation. The well is put into operation in the S4 section, with a buried depth of 1,800 meters, a reservoir depth of 50 meters $^{\circ} \mathrm{C}$, degassing viscosity of $15,853 \mathrm{mpa} \cdot \mathrm{s}$, and freezing point of $41.8^{\circ} \mathrm{C}$. It was put into production in February 2004 with the conventional thickening method. In the initial stage, the daily liquid production was 16.8 tons; the daily oil production was 15 tons; and the water cut was $19 \%$. However, the production decline was fast. Before the test, the daily liquid production was 5 tons; the daily oil production was 0.3 tons; and the water cut was $92.2 \%$. The total accumulated oil was 9,600 tons, and the accumulated water was 15,411 tons.

Implemented in June 2018, a total of $500 \mathrm{t}$ oil displacement system was injected into three slugs: the first slug was filled with $100 \mathrm{t}$ liquid, concentration was $8 \%$, injection displacement was $30 \mathrm{t} / \mathrm{h}$, plug removal and near well space release. In the second slug, 200 tons of liquid is prepared; the concentration is $5 \%$; and the injection displacement is $30 \mathrm{t} / \mathrm{h}$. The reservoir is cleaned, and the liquid outlet channel is improved. The third slug is 200 tons; the concentration is $3 \%$; and the injection displacement is $30 \mathrm{t} / \mathrm{h}$. The oil production curve of well Luo 321-2 is shown in Figure 9. After the implementation, the peak production of daily liquid is 10.7 tons; daily oil is 2.6 tons; water cut is $75.2 \%$; stable production of daily liquid is 4.5 tons; daily oil is 1.4 tons; the effect of the measures lasts for 400 days; the cumulative oil increase is 883 tons; the purpose of dredging seepage channel, reducing pour point, and viscosity is achieved; and the continuous and stable production is realized.

From 2018 to 2020, the technology has carried out 30 field tests in Luo 321, Luo 36, Chen 371, and other blocks of Hekou Oil Production Plant of Shengli Oilfield, with a cumulative oil increase of 1,027,400 tons and an average single well oil increase of 342.5 tons. Compared with steam huff puff, the measure cost is reduced by more than $60 \%$, and the economic benefit is remarkable.

\section{Conclusion}

(1) The viscosity and freezing point of crude oil in the Luojia oilfield are closely related to the content, composition, and proportion of heavy components. 
The larger the molecular weight of asphaltene, gum, and wax, the stronger the intermolecular interaction of heavy components, and the higher the viscosity and wax precipitation point, pour point, and freezing point of crude oil. The viscosity of high pour point and high viscosity oil is mainly affected by asphaltene; the wax precipitation point and pour point are mainly affected by wax; and the freezing point is affected by both of them.

(2) The efficient oil displacement system formed by POA-VA and DBD-DOPAMA was screened. The results show that PD-7 (POA-VA 40\%, DBDDOPAMA 50\%, and P-10C 10\%) is the best flooding system. When the concentration is $10 \%$, the viscosity reduction rate reaches $95.2 \%$, and the freezing point can be reduced by $10.2^{\circ} \mathrm{C}$. The indoor evaluation shows that the oil sample has excellent adaptability, salinity resistance, and temperature resistance, and the oil washing rate can reach more than $60 \%$.

(3) Field application was carried out in well Luo 321-2. A total of 500 tons of the oil displacement system with different concentrations were injected into three slugs. The effect of the measures lasted for 400 days, and the cumulative oil increase was 883 tons. Thirty field tests were conducted in different blocks, and the cumulative oil production increased by 10,274 tons, and the cost of measures was reduced by more than $60 \%$ compared with steam huff puff.

\section{Data Availability}

The data used to support the findings of the study are available from the corresponding author upon request.

\section{Conflicts of Interest}

The authors declare that they have no conflicts of interest.

\section{Acknowledgments}

This work was supported by "The National Natural Science Foundation of China" under Grants 51722406, 61573018, and 51874335; "Shandong Provincial Natural Science Foundation" under Grant JQ201808; "the Fundamental Research Funds for the Central Universities" under Grant 18CX02097A; "the National Science and Technology Major Project of China" under Grant 2016ZX05025001-006; and Sinopec Science and Technology Project under Grant P20050-1.

\section{References}

[1] W. Ding, X. Xing, and T. Yu, "Study on the properties of hydroxysulfobetaine suitable for high temperature and high salt reservoirs," Oil Field Chemical, vol. 30, no. 4, pp. 561-564, 2013.

[2] J. Hou, C. Wu, and F. Zhao, "Optimization and performance evaluation of composite flooding system for high temperature and high salt reservoirs," Petroleum Geology and Development in Daqing, vol. 33, no. 2, pp. 121-126, 2014.
[3] J. Zhao, J. Fang, and D. Zhou, "Tazhong 402CIII Surfactant flooding in high temperature and high salt reservoir," Oilfield Chemistry, vol. 31, no. 3, pp. 405-409, 2014.

[4] G. Zhao, R. Jin, and S. Li, "Performance and application of betaine surfactant TCJ-5 in high temperature and high salt reservoir," Journal of Northeast Petroleum University, vol. 39, no. 3, pp. 111-117, 2015.

[5] Y. Cao, S. Guo, and P. Tang, "Preparation and application of heavy oil emulsion viscosifier S-5," Fine Petrochemical Industry Progress, vol. 5, no. 10, pp. 18-21, 2004.

[6] Q. Tang, "Application of ZDT emulsion viscosity reducer in development of heavy oil in ultra-deep wells," Petroleum Drilling Techniques, vol. 36, no. 2, pp. 74-76, 2008.

[7] F. Wang, S. Wang, and C. Li, "Application of heavy oil viscode-reducing agent HP in well S66 of tahe oilfield," Oilfield Chemistry, vol. 20, no. 3, pp. 210-212, 2003.

[8] F. Yang, S. Xiao, and B. Yao, "Effect of poly (octadecyl acrylate) -vinyl acetate comb-type binary copolymer on crystallization and rheological properties of waxy crude oil," Chemical Journal of Chinese Universities, vol. 37, no. 7, pp. 1395-1401, 2016.

[9] X. Xu, Z. Wei, Q. Ji, C. Wang, and G. Gao, “Global renewable energy development: influencing factors, trend predictions and countermeasures," Resources Policy, vol. 63, no. 10, Article ID 101470, 2019.

[10] X. Xu, C. Wang, and P. Zhou, "GVRP considered oil-gas recovery in refined oil distribution: from an environmental perspective," International Journal of Production Economics, vol. 235, Article ID 108078, 2021. 